\title{
La depuración de aguas residuales. Estudios de soluciones para el municipio de Ambato. Ecuador.
}

The purification of wastewater. Studies of solutions for the municipality of Ambato. Ecuador.

Santiago Andrés Ortiz Montero. ${ }^{1}$, Galo Wilfrido Núnez Aldás. ${ }^{2}$, Alex Gustavo López Arboleda. ${ }^{3} \&$ Alex Xavier Frías Torres. ${ }^{4}$

\begin{abstract}
DOI: $\underline{\text { https://doi.org/10.33262/concienciadigital.v4i3.1803 }}$

Introduction. The quality of wastewater treatment is essentially important for the protection of the environment and the survival of life on the planet. In the city of Ambato. Ecuador does not have an adequate strategy and technology that meets the parameters established by the regulatory entity for these purposes. Objective Provide a proposal for a solution containing the design of a station for the purification and improvement of the quality of wastewater in the city of Ambato. Methodology. The methodology was

\footnotetext{
${ }^{1}$ Master Universitario en Sistemas de Ingeniería Civil, Universidad Técnica de Ambato, C.C 1804662078, Provincia de Tungurahua, correo: sant_ortiz@hotmail.com, ORCID: https://orcid.org/0000-0003-14046467

2 Magister en Docencia y Currículo para la Educación Superior, Universidad Técnica de Ambato, Facultad ingeniería Civil y Mecánica C.C 1802229227, Provincia de Tungurahua, correo: gw.nunez@uta.edu.ec, ORCID: https://orcid.org/0000-0001-7087-1213

3 Master en Vías Terrestres Universidad Técnica de Ambato, Facultad Ingeniería Civil y Mecánica C.C 1802919322, Provincia de Tungurahua, correo: ag.lopez@uta.edu.ec, ORCID: https://orcid.org/00000001-9914-4925

${ }^{4}$ Master en Sistemas de Gestión Ambiental Universidad Técnica de Ambato, Facultad ingeniería Civil y Mecánica C.C 1804159554, Provincia de Tungurahua, correo: ax.frias@uta.edu.ec, ORCID: https://orcid.org/0000-0002-7433-819X.
} 
descriptive, prospective diagnosis, accompanied by theoretical and empirical methods. Results. 1. The design of a station for the purification of wastewater in the city of Ambato is provided. Ecuador; Its characteristics allow to guarantee its reliability and usefulness in solving the problem under study. 2. The wastewater treatment processes differ in each case, given their differences in origin-effluents, agricultural drains, municipal or industrial discharge, implying a correct selection of the technology to be used. 3. It should be planned to incorporate sludge treatment into wastewater treatment, making up the comprehensive set of water and sludge treatment. 4. The effectiveness of the Biodisco alternative is evident, constituting the solution with the best performance in terms of technologies and benefits. Conclusions. The selection of the technology to be used in wastewater treatment plants must be coupled with environmental, economic and longterm sustainability concepts, as an essential component for compliance with the parameters established by the regulatory entity for the protection of the environment. environment and quality of life on the planet. 2. Among the most effective solutions with the best performance, the Biodiscos solution alternative should be evaluated, due to its high degree of technological effectiveness and its contribution to the practical solution.

Keywords: wastewater, purification, wastewater treatment process.

\section{Resumen}

Introducción. La calidad en la depuración de las aguas residuales, es esencialmente importante para la protección del medio ambiente y la supervivencia de la vida en el planeta. En la ciudad de Ambato. Ecuador, no se cuenta con una estrategia y tecnología adecuada que cumpla con los parámetros establecidas por la entidad reguladora para estos fines. Objetivo. Aportar una propuesta de solución contentiva del diseño de una estación para la depuración y mejoramiento de la calidad de las aguas residuales en la ciudad de Ambato. Metodología. La metodología fue descriptiva, diagnóstica de corte prospectivo, acompañada de métodos teóricos y empíricos. Resultados. 1. Se aporta el diseño de una estación para la depuración de las aguas residuales en la ciudad de Ambato. Ecuador; sus características permiten avalar su confiabilidad y utilidad en la solución del problema objeto de estudio.2. Los procesos de tratamiento de las aguas residuales, difieren en cada caso, dado sus diferencias de origen-efluentes, de drenes agrícolas, descarga municipales o industriales, implicando una correcta selección de la tecnología a emplear. 3. Se debe prever incorporar al tratamiento de aguas residuales, la depuración del lodo, conformando el conjunto integral de tratamiento de agua y lodo.4. Resulta evidente la efectividad de la alternativa de Biodisco, constituyendo la solución con mejor rendimiento en cuanto a tecnologías y beneficios. Conclusiones. La selección de la tecnología a utilizarse en las plantas depuradoras de aguas residuales, debe estar acoplada con las concepciones ambientales, económicas y de sostenibilidad a largo plazo, como componente esencial para el cumplimiento de los parámetros establecidos por la entidad reguladora para la protección del medio ambiente y la calidad vida en el planeta. 2. Dentro de las soluciones más efectivas y con mejor rendimiento se debe evaluar la alternativa de solución de 
Biodiscos, debido a su alto grado de efectividad tecnológica y su contribución a la solución práctica.

Palabras claves: aguas residuales, depuración, proceso de tratamientos de aguas residuales.

\section{Introducción}

La contaminación del agua plantea amenazas para la salud y la calidad de vida. La falta de agua de buena calidad perjudica tanto al medio ambiente como al medio acuático y por consiguiente la calidad de vida en el planeta. Se coincide con autores precedentes que, si no se logra un adecuado tratamiento de las aguas contaminadas, la descomposición de la materia orgánica y compuestos amoniacales del agua residual, originaria el consumo de oxígeno disuelto en el agua, por lo cual se produciría malos olores, habría un elevado número de patógenos e incluso aumentaría la eutrofización de las aguas causado por los altos contenidos de fosforo y nitrógeno.

Según plantean Raschid \& Jayakody (2008), las aguas residuales constituyen una combinación de efluentes domésticos, conformadas por aguas negras- constituidas de excremento, orina y lodos fecales; aguas grises, que incluyen aguas servidas de lavado y baños; agua de locales comerciales e instituciones, incluidos hospitales; efluentes industriales, aguas pluviales y otras escorrentías urbanas; y escorrentías agrícola, hortícola y acuícola.

Las consecuencias al medio ambiente que se genera por la falta de tratamiento de aguas residuales, han sido expuestas por diversos autores; al respecto Vázquez, (2017), explica que la Toxicidad: afecta directamente a la flora y la fauna de los cuerpos de agua que recepcionan el agua contaminada y a la persona que la manipule, mientras que las Infecciones: se refieren a los diferentes organismos patógenos que son transmitidos mediante el agua, que afectan a los organismos vivientes con los que entran en contactos, a la Contaminación Térmica de las reservas de agua: Los residuos líquidos industriales tienden a aumentar la temperatura de las mismas, Malos Olores: las bacterias y elementos que contienen las aguas residuales producen gases debido a su proceso de descomposición.

Refiriéndonos al Informe Mundial de las Naciones Unidas sobre el Desarrollo de los Recursos Hídricos (2017), los países desarrollados tratan en alrededor del 70\% las aguas residuales municipales e industriales que generan, los países en vías de desarrollo lo hacen en un $28 \%$, mientras que en los países subdesarrollados solo el $8 \%$ reciben algún tratamiento.

En Ecuador, al igual que en otros países de Latinoamérica, los recursos hídricos como ríos, quebradas, mares entre otros reciben todo tipo de aguas residuales. Al respecto el Reporte de Estadística de Información Ambiental Económica de Agua y Alcantarillado, elaborado por el Instituto Nacional de Estadística y Censos (INEC) de Ecuador en el año 
2015, reflejó que, en el sector urbano del país, el 38,14\% no realizan tratamiento de sus aguas residuales.

Esto ha provocado que actualmente el $70 \%$ de los ríos ecuatorianos estén en condiciones críticas de contaminación, especialmente los ríos que están ubicados en las principales ciudades del país. Frente a esto algunas ciudades como Quito y Guayaquil han implementado programas para la descontaminación de ríos.

Sin embargo, en otras ciudades medianas aún no se aplica un mejoramiento oportuno y adecuado para el tratamiento de las aguas residuales como es el caso de la ciudad de Ambato, siendo esta precisamente la ciudad objeto de estudio.

Considerando la situación problémica existente en la ciudad de Ambato. Ecuador; dada precisamente entre otros aspectos por el no cumplimiento del Decreto Ejecutivo 3516 del 2003, Texto Unificado Legislación Secundaria del Ministerio del Ambiente, conocido como Libro IV (TULAS), en cuanto a los límites establecidos para la depuración de las aguas residuales y la necesidad de buscar soluciones que tributen a mejorar la calidad de estas, el presente artículo centra su objetivo en: Aportar una propuesta de solución contentiva del diseño de una estación para la depuración, tratamiento y mejoramiento de la calidad de las aguas residuales en la ciudad de Ambato. Ecuador. Para ello se parte de datos proporcionados por la entidad encargada de las depuradoras, la Empresa Municipal de Agua Potable (EMAPA), de la ciudad de Ambato. Ecuador.

Un acercamiento a las generalidades del proceso de depuración de las aguas residuales, la selección de la tecnología y su marco legal en el contexto internacional y nacional.

La depuración, tratamiento y mejoramiento de la calidad de las aguas residuales tanto a nivel internacional como nacional ocupa un tema esencialmente importante en la actualidad; máxime si tenemos presente su valor e importancia no solo para la protección del medio ambiente, sino también para la supervivencia de las distintas especies en la tierra, incluyendo la vida humana.

En el caso de Ecuador el tratamiento de las aguas residuales está regulado por el Decreto Ejecutivo 3516 del 2003, Texto Unificado Legislación Secundaria del Ministerio del Ambiente, Libro IV (TULAS), con última vigencia y actualización en el 2015. En su Artículo 216: referente al tratamiento de aguas residuales rurales y urbanas, indicando que el mismo tiene como objeto la Prevención y Control de la Contaminación Ambiental, en lo relativo al recurso agua determinando los límites permisibles, disposiciones y prohibiciones para las descargas en cuerpos de aguas o sistemas de alcantarillado.

De acuerdo con Noyola, Morgan, Guereca (2013), la principal finalidad del tratamiento del agua residual es remover el material contaminante del agua, orgánico e inorgánico, que puede estar conformado en forma de partículas en suspensión y/o disueltas, de manera se pueda lograr un agua con la calidad requerida por la normativa de descarga vigente o que cumpla con el tipo de reutilización a la que se destinará. 
En esta dirección Rodríguez, García, \& Pardo (2015), exponen que las: Estaciones Depuradoras de Aguas Residuales Municipales (EDAR), son utilizadas en la remoción de contaminantes presentes en el agua residual cruda, y responden a una elevada variabilidad temporal del flujo o caudal de entrada, así como a la variabilidad de las concentraciones o componentes propios de estas aguas residuales. Al respecto, autores como Hong, (2003) \& Bdour, (2009), han señalado que para lograrlo se requiere de una interacción en los mecanismos biológicos, físicos y químicos entre los procesos unitarios, los fenómenos hidrodinámicos y la adaptabilidad.

Es debido a lo anterior que, para el dimensionamiento y diseño de las EDAR, Gernaey, (2004), definió elementos básicos para su dimensionamiento y diseño, tales como la caracterización del agua residual cruda, la finalidad del modelo de la planta, el sistema hidráulico, el análisis del modelo en estado estacionario, la calibración del modelo, y otros.

En este sentido, Metcalf \& Eddy, (2003); APHA, (2009), Hernández, (1996); Crites, (2000), Villaseñor, (2001) y Hammer, (2012); han indicado que la composición típica de las aguas residuales crudas es una de las variables principales a tener en cuenta para la selección de una EDAR, ya que sean estas concentraciones fuertes, medias, débiles o ligeras, dependerá el grado de tratamiento, la tecnología a utilizar y la eficiencia o rendimiento que se desea para cumplir con las normas aprobadas. No obstante, también hay que considerar los aportes de Schoenberger, (2009), al señalar que la mejor tecnología a utilizarse en plantas depuradoras de aguas residuales, debe estar acoplada con las concepciones ambientales, económicas y de sostenibilidad a largo plazo.

Lo cierto es que, la selección de la tecnología más conveniente para una estación depuradora de aguas residuales municipal, sigue siendo un tema de debate por lo que distintos autores entre los que destaca Chung (2013), han definido que en su selección se hace necesario tener en cuenta varios aspectos de evaluación, tales como: la caracterización del agua residual cruda o influente, la tecnología apropiada al ambiente local, el cumplimiento de la normativa vigente, la evaluación de factores ambientales externos a la planta, la evaluación de la viabilidad económica y la evaluación de factores tecnológicos de operación y mantenimiento.

Por su parte Rodríguez, García, \& Pardo (2015), han puntualizado que, de manera concreta, para ubicar una planta de tratamiento debe cumplirse con elementos espaciales para la óptima selección del sitio, debido a la racionalización que ello se deriva, referente a la ubicación de recursos naturales, el empleo de energía y la minimización de los posibles efectos ambientales, como son los olores, ruidos, espumas, en el área de estudio o de intervención.

En esta misma dirección Benujah, (2013), explica que las aguas residuales se generan partiendo de actividades de tipo doméstico, industrial, comercial, institucional, agrícola y municipal, por lo que la disposición de estas aguas residuales en cuerpos de aguas receptores implica necesariamente de una ubicación espacial 
En correspondencia a ello, Sánchez' (2017), ha remarcado que debe ser considerado que los procesos de tratamiento de aguas residuales difieren en cada caso, dado sus diferencias de origen-efluentes de drenes agrícolas, descarga municipales o industriales, lo que requiere una correcta identificación de las tecnologías más convenientes a utilizar, una vez realizado una cuantiosa cuantificación de los volúmenes de descarga y de las características físicas, químicas y biológicas del agua a tratar.

Noyola, Morgan, Guereca, (2013), han referido que el nivel de tratamiento para las aguas residuales, dependerá de la utilización o disposición final del agua tratada. Estos niveles están concebidos a los siguientes: Tratamiento Preliminar, concernientes a la eliminación de aquellos componentes que puedan provocar problemas operacionales y de mantenimiento en el proceso de tratamiento o en los sistemas auxiliares tratamiento preliminar de un agua residual, al Tratamiento Primario, en la que una porción de sólidos y materia orgánica suspendida es removida del agua residual utilizando la fuerza de gravedad como principio, al Tratamiento Secundario, donde se elimina la materia orgánica biodegradable (principalmente soluble) por medios preferentemente biológicos, donde los contaminantes presentes en el agua residual, son transformados por los microorganismos en materia celular, energía para su metabolismo y en otros compuestos orgánicos e inorgánicos, y finalmente al Tratamiento Terciario, con la finalidad de eliminar compuestos solidos suspendidos, nutrientes y materia orgánica remanente no biodegradable. Este tratamiento se realiza cuando deben ser cumplidas condiciones de descargas estrictas del agua o si el agua tendrá determinada especificidad de uso.

Un aspecto a tener en cuenta es el relacionado con los que se denominan contaminantes orgánicos emergentes (COEs), o nuevos contaminantes, los que, según autores como Rubio, Chica, Peñuela, (2013), incluye sustancias bioacumulables, persistentes y tóxicas; tales como productos de higiene y cuidado personal, productos farmacéuticos, drogas de abuso, surfactantes, aditivos industriales, y una gran variedad de compuestos químicos.

Figura No. 1- Esquema de la línea de agua residual de una Estación de Aguas residuales (EDAR)convencional.

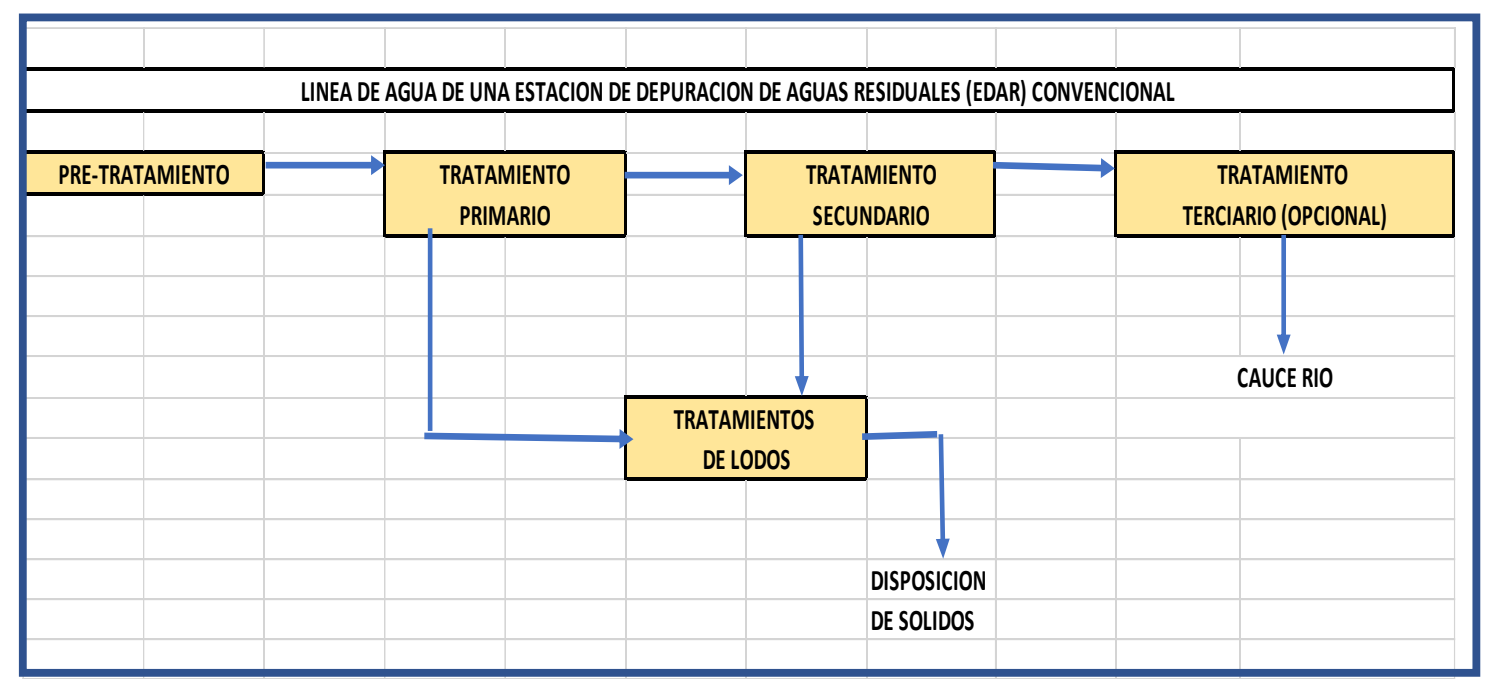


Estos mismos autores afirman que los sistemas de aguas residuales producen lodos, que deben ser tratados para utilizarlos adecuadamente. De igual manera indican en la Figura 2, los diferentes sistemas de tratamiento de aguas residuales, así como señalan 5 sistemas de tratamientos de lodos, los que deben ser incorporados a los sistemas de tratamiento de agua residual, conformando el conjunto integral de tratamiento de agua y lodos.

Figura 2- Tecnologías para el tratamiento de agua residual y lodo.

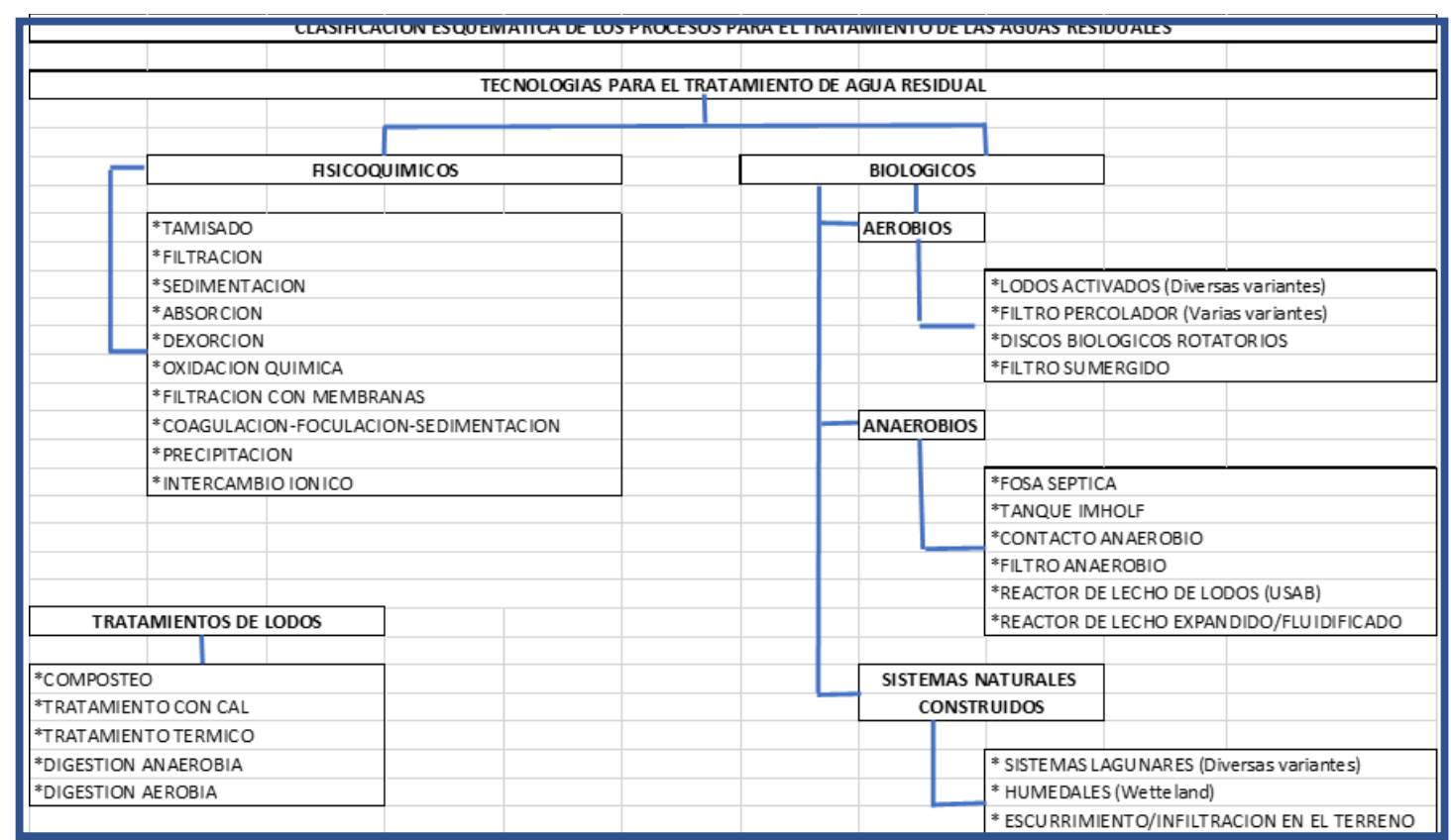

Precisamente en esta misma idea Petrovic et al. (2003), Andreozzi et al. (2003) han señalado que una de las principales fuentes de entrada de estos nuevos contaminantes al medio ambiente, están dados especialmente por las aguas residuales no tratadas y los efluentes que proceden de estaciones depuradoras de aguas residuales, que en la actualidad no han sido diseñadas para tratar este tipo de contaminantes.

En atención a lo expuesto Dougherty et al. (2010), remarca que, como consecuencia de ello una proporción de estos compuestos entran con una gran toxicidad al medio acuático, alterando acuíferos y sistemas fluviales y marinos entre otros.En relación a las aguas residuales, Vilanova, et al, (2017), han afirmado que la caracterización de estas aguas residuales urbanas, se realiza teniendo en cuenta una serie de parámetros que son utilizados para medir el grado de contaminación y a su vez para realizar el diseño de las estaciones depuradoras de aguas residuales, dado que, en función del grado de contaminación existente en una zona urbana específica, requerirán diferentes niveles de tratamiento.

Estos autores aseveran que los parámetros frecuentes para medir el grado de contaminación de las aguas residuales son: la Materia Orgánica (MO), que expresa los compuestos orgánicos del carbono, representando la capacidad de absorción del oxígeno disuelto que contienen las aguas naturales, los Solidos en Suspensión (SS), que cuantifica el impacto se los sólidos en el cauce receptor, cuya acumulación origina la turbidez y la formación de fangos y el Nitrógeno (N/ mgN/l) Total y Fosforo (P/mgP/l), que ofrecen 
una medida de nutrientes encargados de la eutrofización, o sea que promueven el crecimiento excesivo de algas y otras plantas. El Nitrógeno expresa el contenido de Nitrógeno Total Kjeldahl (KTN).

De igual manera alegan que para la medición de aguas residuales de una población se usa el concepto de habitante-equivalente (h-e), que constituye una unidad de medición de la contaminación en las aguas residuales urbanas.

En función de esto expresan los límites de contaminación establecidos en España, para el afluente de una estación depuradora de aguas residuales, que se recogen en la siguiente tabla:

Tabla No. 1-Limites de vertido en aguas residuales urbanas

\begin{tabular}{lc}
\hline \multicolumn{1}{c}{ Parámetro } & Limite en efluente \\
\hline Sólidos en suspensión $(\mathrm{mg} / \mathrm{l})$ & 35 \\
$\mathrm{DBO5}(\mathrm{mgO} 2 / \mathrm{l})$ & 25 \\
$\mathrm{DQO}(\mathrm{mgO} 2 / \mathrm{l})$ & 125 \\
Nitrógeno $(\mathrm{mgN} / \mathrm{l})$ & $15(10 \mathrm{~K} \mathrm{a} 100 \mathrm{~K} \mathrm{~h}-\mathrm{e})$ \\
& $10(\geq 100 \mathrm{~K}$ h-e $)$ \\
Fosforo $(\mathrm{mgP} / \mathrm{l})$ & $2(10 \mathrm{~K}$ a $100 \mathrm{~K} \mathrm{~h}-\mathrm{e})$ \\
& $1(\geq 100 \mathrm{~K} \mathrm{~h}-\mathrm{e})$ \\
\hline
\end{tabular}

Fuente: Norma Española del Medio Ambiente.

En Ecuador, conforme al Registro Oficial Edición Especial 387 de 04-nov.-2015Reforma Texto Unificado Legislación Secundaria, Medio Ambiente, Libro VI, decreto ejecutivo 3516. Anexo 1: norma de calidad ambiental y de descarga de efluentes al recurso agua, se recoge en la página 21, articulo 5.2.4.7, la Tabla 9- Limites de descarga a un cuerpo de agua dulce, los siguientes parámetros, que corresponden a valores medios diarios:

Tabla No. 2- Limites de descarga a un cuerpo de agua dulce en Ecuador.

\begin{tabular}{lc}
\hline \multicolumn{1}{c}{ Parámetro } & Limite en efluente \\
\hline Sólidos en suspensión $(\mathrm{mg} / \mathrm{l})$ & 130 \\
DBO5 (mgO2/l) & 100 \\
DQO (mgO2/l) & 200 \\
Nitrógeno Total Kjeldahl (mg/l) & 50 \\
Fosforo Total (mg/l) & 10 \\
\hline
\end{tabular}

Fuente: Norma de calidad ambiental y de efluentes de descarga a un cuerpo de agua dulce.Ecuador.

En el documento se enuncia además que, para determinar los valores y concentraciones de los parámetros determinados en esta Norma oficial ecuatoriana, se deberá aplicar los

métodos establecidos en el manual "Standard Methods for the Examination of Water and Wastewater", así como también deberán considerarse las siguientes Normas del Instituto Ecuatoriano de Normalización (INEN): Norma Técnica Ecuatoriana NTE INEN 2169:98. Agua: Calidad del agua, muestreo, manejo y conservación de muestras y Norma Técnica Ecuatoriana NTE INEN 2176:98. Agua: Calidad

En este contexto, Montes (2015), señaló que la valoración de las características de un agua residual puede realizarse de diferentes maneras, en función de un propósito 
especifico, no obstante es importante remarcar que toda caracterización de aguas residuales implica un programa de muestreo apropiado que asegure la representatividad de la muestra así como la realización de un análisis de laboratorio de conformidad con las normas aprobadas, que asegure la precisión y la exactitud de los resultados.

Por su parte se comparte con Rico, Arahuetes, Morote (2016), quienes subrayaron las grandes ventajas que reporta la reutilización de las aguas residuales depuradas urbanas, las cuales además del beneficio ambiental que representa, han señalado que la reutilización de aguas residuales constituye una fuente alternativa de extraordinario interés para paliar la escasez natural de agua, los efectos de intensas sequías, y las posibles consecuencias que tendría el cambio climático.

Igualmente, el uso de aguas regeneradas contribuye ya a satisfacer consumos agrícolas, urbanos y turísticos, reduciendo así el consumo de agua potable durante situaciones de sequía. Es por ello que la reutilización de aguas residuales constituye un recurso alternativo de vital importancia en la actualidad; aunque podría incrementarse todavía más su nivel de aprovechamiento atendiendo la demanda de determinados usos, que actualmente consumen agua potable.

Guardino, González (2012), señalan que en evaluaciones realizadas se ha constatado que los proyectos de depuración de aguas residuales han presentado una serie de deficiencias en lo relativo a la inclusión de criterios de sostenibilidad en los mismos, indicando que los proyectos de depuración no están relacionados con el ciclo urbano del agua y tampoco con otros ciclos como el de los residuos, cuando una EDAR es una importante productora de los mismos.

Los mismos autores han indicado que en el futuro debería contemplarse la EDAR de manera integral, precisándose que el proyecto debe estar englobado en una Evaluación Ambiental Estratégica y dentro de un ciclo del agua abierto al resto de ciclos de materia y energía. Además, enfatizan en que los proyectos deberían incluir un estudio previo de alternativas de tecnologías a utilizar, dado que estos estudios, en la práctica, no se realizan. De igual manera, compartimos que dado la elevada incidencia que tiene la energía en la instalación de las estaciones de depuración de aguas residuales, se hace muy necesario incluir la optimización energética de las mismas en el proyecto.

\section{Alternativas para mejorar el tratamiento de aguas residuales: Una experiencia en Ambato.}

El proyecto aborda las posibles alternativas para mejorar el tratamiento de la calidad de aguas residuales debido a que las depuradoras actuales ubicadas en el municipio de Ambato, no cumplen con los límites permisibles establecidas por el TULAS.

Se parte de datos proporcionados por la entidad encargada de las depuradoras, la Empresa Municipal de Agua Potable (EMAPA). Las características del agua son datos tomados de tres depuradoras en funcionamiento, las cuales van a ser remplazadas con una de las dos alternativas planteadas en este proyecto.

- Diagnóstico del área de estudio

- Ubicación del área de estudio 
Las plantas de tratamiento de aguas residuales a rediseñar están localizadas a $2580 \mathrm{msnm}$, en el sector de Techo Propio, las cuales están encargadas por la Municipalidad de Ambato, Ecuador.

- Situación actual de las EDAR.

La EDAR de Techo P1 y Techo P3 están localizadas a 2517 y $2587 \mathrm{msnm}$, las cuales constan como línea de agua de pretratamiento (aliviadero, cribador por rejas, desarenador, trampas de grasas), que pasa al tratamiento primario que es por medio de una fosa séptica, de ahí continúa al tratamiento secundario anaerobio y finaliza con un tratamiento terciario que es por desinfección con cloro. La línea de fangos es por medio de eras de secado.

La EDAR de Techo P2 está localizada a $2550 \mathrm{msnm}$, la cual constan como línea de agua de pretratamiento (aliviadero, cribador por rejas, desarenador, trampas de grasas), que pasa al tratamiento primario que es un proceso físico - químico de ahí continúa al tratamiento secundario que es a través del reactor UASB y finalizando con un tratamiento terciario que es por desinfección con cloro. La línea de fangos es por medio de eras de secado.

\section{- Problema existente}

El problema que existe en la ciudad de Ambato se concreta en que las plantas existentes, no cumplen con los límites permisibles de vertido al río, violándose los parámetros establecidos por la entidad reguladora. Por lo que se requiere buscar alternativas de solución

- Tamaño de la población

El sector de Techo Propio cuenta con una población de 13540 habitantes, de los cuales 7217 son mujeres $(53.3 \%)$ y 6323 son hombres $(46,7 \%)$; según datos obtenidos en el censo realizado en los talleres de diagnóstico en cada una de las comunidades de la parroquia y el censo realizado por el INEC en el año 2010.

- Climatología

El clima de Ambato se clasifica como cálido y templado. Es una gran cantidad de lluvia en Ambato, incluso en el mes más seco. El clima aquí se clasifica como Marítimo de costa occidental $(\mathrm{Cfb})$ por el sistema Köppen-Geiger. La temperatura promedio en Ambato es $14.6^{\circ} \mathrm{C}$. La precipitación es de $504 \mathrm{~mm}$ al año

- Tipo de Suelo

El sector de Techo Propio posee una topografía irregular, ligeramente inclinada hacia el Río Ambato, conformado por suelos pocos profundos erosionados y sobre una capa dura cementada a menos de $0,5 \mathrm{~m}$ de profundidad. En su mayoría estos suelos son fértiles, de textura franco a franco-arenoso, con buena retención de humedad.

- Datos de Partida 
Se procede, teniendo en cuenta que previo al diseño de una estación depuradora de aguas residuales deben ser definidas las características que tiene el agua bruta de entrada, tanto sus niveles de contaminación, así como establecer los objetivos finales, tanto para la línea de agua como para la de fango.

- Caudales de Partida

- Caudal Medio

Partiendo del tamaño de la población estudiada y teniendo en cuenta las tres PTAR actuales se pudo establecer un caudal medio de entrada.

Tabla No 3- Caudal medio total de las 3 EDAR existentes.

\begin{tabular}{cc}
\multicolumn{2}{c}{ Caudal medio Total } \\
\hline \multicolumn{2}{c}{ CAUDALES } \\
\hline PTAR & Q medio \\
\hline Techo P1 & $27,18 \mathrm{~m} 3 / \mathrm{h}$ \\
Techo P2 & $30,82 \mathrm{~m} 3 / \mathrm{h}$ \\
Techo P3 & $21,28 \mathrm{~m} 3 / \mathrm{h}$
\end{tabular}

- Caudal Punta

Fue necesario estimar la caudal punta para poder sobredimensionar la planta depuradora en caso de producirse picos en la entrada del caudal, asegurándose a futuro el correcto funcionamiento de dicha planta. Las previsiones se realizaron de forma que no se produzcan bypass de las aguas residuales en la red de alcantarillado ni en el interior de la planta de tratamiento, por este motivo se proyectan pozos aliviaderos. Para el respectivo cálculo y conforme a García, Betancourt y Salas (2016), se usó la Formula 1 tomándose la suma de los tres caudales medios de las tres depuradoras a estudiar.

Fórmula 1: $Q p=Q m *(1,5+2,5 \sqrt{ } Q m)$

- Caudal Máximo

El caudal máximo, se pudo determinar a partir de una serie de fórmulas matemáticas de tipo empírico, y de acuerdo a García, Betancourt y Salas (2016), aplicándose la Formula 2.

Fórmula No. 2: $\quad Q m a ́ x=Q m *(1,15+2,575(Q m) 0,25$

Tabla No 4- Caudal máximo y de punta de las EDAR.

\begin{tabular}{lcc}
\hline \multicolumn{3}{c}{ CAUDALES } \\
\hline \multicolumn{3}{c}{ PTAR } \\
\hline Q punta & 141,16 & $\mathrm{~m} 3 / \mathrm{h}$ \\
Q máx. & 159,57 & $\mathrm{~m} 3 / \mathrm{h}$ \\
\hline
\end{tabular}

- Niveles de contaminación de partida. 
En relación a la carga contaminante del agua, se tomaron los datos proporcionados por la Municipalidad de Ambato, encargada de las EDAR, información que se recoge en la Tabla No 5.

Tabla No 5- Concentración de partidas (Datos de entrada a la EDAR).

\begin{tabular}{lccc}
\hline \multicolumn{4}{c}{ Concentración de partidas (Datos de entrada a la EDAR) } \\
\hline \multirow{2}{*}{ CARGAS } & TP1 & TP2 & TP3 \\
& $\mathrm{mg} / \mathrm{l}$ & $\mathrm{mg} / \mathrm{l}$ & $\mathrm{mg} / \mathrm{l}$ \\
DBO5 & 388,67 & 682,67 & 382 \\
DQO & 529 & 1327,33 & 788 \\
Solidos suspendidos & 218,33 & 331,67 & 371,67 \\
Nitrógeno & 36,8 & 70,00 & 55,20 \\
Fosforo & 4,92 & 5,34 & 3,82 \\
\hline \multicolumn{4}{c}{} \\
\hline Q total m3/d & 652,32 & 739,58 & 510,62 \\
\hline
\end{tabular}

Para el cálculo de los kilos/día de las concentraciones se procedió a multiplicar el caudal por la carga, conforme a la Formula Nro 3.

Fórmula No 3: $\quad$ Carga $\mathrm{Kg} / \mathrm{d}=$ QTotal $\mathrm{m} 3 \mathrm{~d} *$ concentración de entrada $\mathrm{mg} / \mathrm{l}$

Tabla No 6- Cargas en $\mathrm{Kg} / \mathrm{d}$ en las EDAR existentes.

\begin{tabular}{lccc}
\hline \multicolumn{4}{c}{ Cargas en Kg/d en las EDAR } \\
\hline \multirow{2}{*}{ CARGAS } & TP1 & TP2 & TP3 \\
& $\mathrm{mg} / \mathrm{l}$ & $\mathrm{mg} / \mathrm{l}$ & $\mathrm{mg} / \mathrm{l}$ \\
\hline DBO5 & 253,54 & 504,89 & 195,06 \\
DQO & 345,08 & 981,67 & 402,37 \\
Solidos suspendidos & 142,42 & 245,3 & 189,78 \\
Nitrógeno & 24,01 & 51,77 & 28,19 \\
Fosforo & 3,21 & 3,95 & 1,95 \\
\hline
\end{tabular}

Para el cálculo de las concentraciones totales se procede a la sumatoria de las cargas de las tres depuradoras dividido entre el caudal total, conforme a la Formula No 4.

Fórmula No 4: $\quad$ Concentración total $m g l=\sum$ Cargas $\mathrm{Kg} d / \sum Q T$ Total $\mathrm{m} 3 \mathrm{~d}$

Tabla No 7- Concentración Total de los parámetros de carga contaminante

\begin{tabular}{lc}
\hline \multicolumn{2}{c}{ Concentración Total } \\
\hline \multicolumn{1}{c}{ Cargas } & Concentración \\
& $\mathrm{mg} / \mathrm{l}$ \\
\hline DBO5 & 501 \\
DQO & 909 \\
Solidos suspendidos & 304 \\
Nitrógeno & 55 \\
Fosforo & 5 \\
\hline
\end{tabular}


- Características del agua depurada

Anteriormente al diseño de la estación, fue necesario establecer los niveles máximos de DBO5, DQO, SST, Nitrógeno y Fosforo del agua depurada. Para fijar estos niveles de contaminación máximos del agua depurada se puntualizaron los parámetros establecidos según la Norma de calidad Ambiental y de descarga de efluentes del Ecuador.

Fue necesario realizar una decantación primaria dado a que utilizando solo un tratamiento físico-químico no cumplía con la salida exigida. Obteniendo finalmente el siguiente rendimiento, que aparece en la Tabla No. 6.

Tabla No 8- Parámetros de contaminación permisible del agua depurada sin decantador primario.

\begin{tabular}{lccc}
\multicolumn{3}{c}{ Parámetros de contaminación permisible del agua depurada sin decantador } \\
primario
\end{tabular}

Lo anterior planteó considerar la realización de un decantador primario seguido de un decantador secundario, de manera que se pudiera cumplir con la exigencia de salida del agua depurada al cauce del río, obteniéndose el siguiente rendimiento, que se refleja en la Tabla No 9.

Tabla No 9- Parámetros de contaminación del agua depurada sin decantador primario.

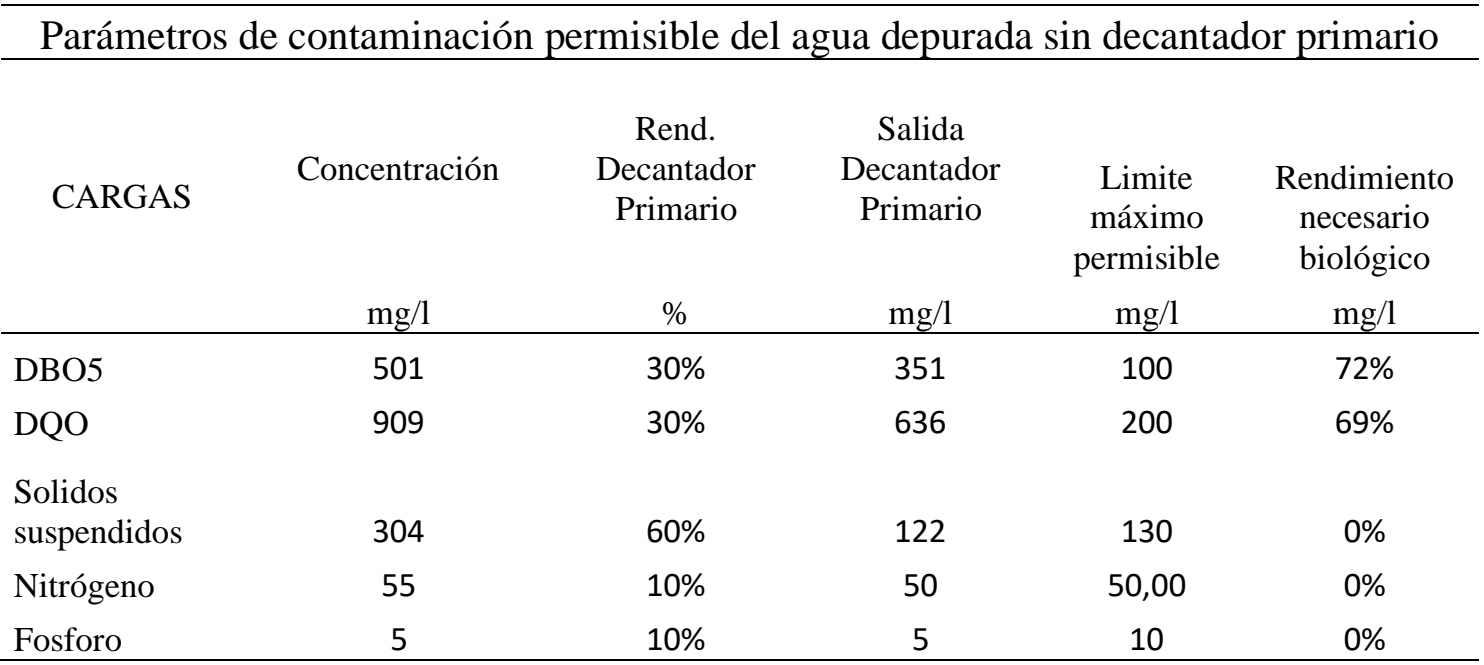

- Posibles alternativas para la depuración de las aguas residuales. 
Una vez establecidos los datos de partida y los objetivos de depuración, se procedió al planteamiento de diferentes procesos para el tratamiento de aguas residuales. Las alternativas propuestas fueron diferentes por su tratamiento secundario, por lo que la mayor parte del proceso, tanto en la línea de agua como en la línea de fango, fueron comunes.

Las alternativas para el tratamiento secundario fueron las siguientes:

- Diseño de Biodiscos

- Diseño de Lechos Bacterianos

- Diseño de un reactor UASB

- Fangos Activos

- Filtro Anaerobio de Flujo Ascendente

Las dos últimas alternativas no fueron diseñadas debido a que los Fangos Activos nos dieron mejores resultados a las sugeridas por los límites permisibles, con un consumo de energía más elevado y una mayor utilización de recursos, pudiéndose alcanzar los límites permisibles necesarios con otras alternativas más convenientes. De igual manera con los filtros anaerobios de flujos ascendentes, existió mucho estancamiento de grasas, aceites y residuos sólidos que se acumularon en el lecho de piedra obstruyendo el filtro, además no se cumplía con los rendimientos necesarios.

Seguidamente se detallaron las características y el funcionamiento de las alternativas, finalizando todas mediante el tratamiento biológico con una decantación secundaria.

Primero se definieron las etapas comunes a todas las alternativas, distinguiendo la línea de agua y la de fango. De igual manera se realizó el cálculo hidráulico.

Los cálculos hidráulicos realizados fueron para determinar la cota de la línea piezométrica a lo largo de toda la planta depuradora. En este caso el cálculo se realizó en el punto de aguas arribas y se continuó aguas abajo, debido a que el río se encuentra en una cota muy baja a la del terreno.

Todos los cálculos fueron basaron en el análisis del comportamiento hidráulico de los distintos elementos que componen los procesos mencionados de la planta depuradora, relacionándose entre ellos mediante las distintas láminas de agua a la entrada y salida de los mismos.

Todas las cotas de este proyecto fueron expresadas en metros sobre el nivel del mar. Los cálculos estuvieron basados en los criterios geotécnicos, topográficos e hidrogeológicos básicos, conformándose los siguientes:

- Cota de vertido

- Cota de entrada al colector

- Caudal máximo en pretratamiento

- Caudal máximo después del pretratamiento

- Caudal de recirculación externa de fangos. 
Los datos de niveles en cada punto permitieron definir la ubicación en alzado de cada elemento del tratamiento, así como la definición de los resguardos necesarios para evitar el desbordamiento en el caso que existiera una punta de caudal no prevista.

- Presupuesto de las diferentes alternativas de las Estaciones de Depuración de Aguas Residuales (EDAR).

Una vez realizados los cálculos técnicos económicos de las alternativas de EDAR evaluadas, se realizó el análisis de presupuesto correspondiente de cada una, lo cual a manera de resumen se recoge en la Tabla No 8.

Tabla No. 10- Presupuesto de las diferentes alternativas de EDAR evaluadas.

\begin{tabular}{ll}
\hline & $\begin{array}{c}\text { Alternativa evaluada } \\
\text { Precio Final }\end{array}$ \\
\hline EDAR con Biodiscos & \\
EDAR con Lechos Bacterianos & $\$ 1.305 .861,96$ \\
EDAR con UASB & $\$ 666.797,31$ \\
\hline
\end{tabular}

\section{Metodología.}

La investigación desarrollada siguió una metodología descriptiva, de corte prospectivo, dado que la información se fue registrando y evaluando conforme ocurrió el estudio metodológico para la selección de la tecnología más adecuada a emplear en la EDAR.

Para ello se contemplaron varias etapas, iniciándose por el diagnóstico de la situación objeto de estudio, que incluyo los datos de partida. Seguidamente se determinaron las alternativas posibles para la depuración de las aguas residuales, una vez precisado el Tratamiento Primario se definieron las alternativas posibles para el Tratamiento Secundario, estableciéndose las siguientes alternativas:

- Diseño de Biodiscos

- Diseño de Lechos Bacterianos

- Diseño de un Reactor UASB

- Fangos Activos

- Filtro Anaerobio de Flujo Ascendente

Posteriormente siguiendo las fases de investigación se determinan las tres posibles alternativas, concretándose en el Diseño de Biodiscos, Diseño de Lechos Bacterianos y en el Diseño de un Reactor UASB

Ello permitió la anticipación a situaciones futuras importantes en la toma de decisiones, en las condiciones de horizonte del proyecto, en la obtención de la calidad del agua residual cruda y tratada, así como en el cumplimiento de las normas ambientales aprobadas vigentes, referente a las descargas de aguas residuales tratadas.

Se utilizaron además métodos teóricos, como el analítico-sintético, inductivo -deductivo y la revisión documental. Dentro de los métodos empíricos empleados se encuentra la 
observación, los cuales favorecieron la obtención de resultados relacionados con la integración de los elementos de investigación necesarios para la elaboración de la metodología de selección de la tecnología adecuada para la implementación de una estación de tratamiento de aguas residuales, que dé solución al problema existente.

\section{Resultados}

Como parte del proceso investigativo desarrollado se llegan a establecer los siguientes resultados:

1. Se logra aportar el diseño de una estación para la depuración, tratamiento y mejoramiento de la calidad de las aguas residuales en la ciudad de Ambato. Ecuador; la misma está precedida por un estudio basado en la revisión científica documental y en la aplicación de experiencias previas que permiten avalar su confiabilidad y utilidad en la solución del problema objeto de estudio.

2. Queda demostrado en las diferentes literaturas consultadas que los procesos de tratamiento de estos tipos de agua, difieren en cada caso, dado sus diferencias de origen-efluentes de drenes agrícolas, descarga municipales o industriales, lo que requiere una correcta identificación de las tecnologías más convenientes a utilizar.

3. Los diferentes sistemas de tratamiento de aguas residuales existentes, producen lodos en su funcionamiento, por lo que los sistemas de tratamientos de lodos, también deben ser incorporados a los sistemas de tratamiento de agua residual, conformando el conjunto integral de tratamiento de agua y lodos.

4. Se evidencia que una de las mejores alternativas de solución y con mejor rendimiento en cuanto a tecnología y beneficios, está relacionado con el proyecto de Biodiscos, debido a que su sencillez implica que las tareas de conservación de la Planta sean muy reducidas, así como el Personal Operario requerido, la misma presentan una buena resistencia a las sobrecargas de materia orgánica, así como a las sobrecargas de materiales o sustancias tóxicas, además la contaminación sonora es prácticamente inexistente teniendo como ventaja que como la construcción de este tipo de plantas es modular, tiene una gran facilidad para ampliar las instalaciones y así tener una mayor eficiencia.

5. Los autores precedentes, han señalado que la composición típica de las aguas residuales crudas municipales, constituye una de las variables a tener en cuenta para seleccionar una EDAR, por lo que incidirá en el grado de tratamiento, la tecnología a utilizar y la eficiencia o rendimiento esperado para cumplir con las normas aprobada de calidad ambiental y de descarga de efluentes al recurso agua en Ecuador, teniendo en cuenta los parámetros permisibles del agua residual, (DBO5, DQO, Solidos suspendidos, Nitrógeno y Fosforo).

6. De igual manera, la generalidad de los autores coincide en los indicadores por tecnologías de tratamiento de las aguas residuales a tener en cuenta, tales como los indicadores de consumo de energía eléctrica, el costo de inversión, de operación y de mantenimiento, el área posible construida, la producción de lodos y la producción de biogas, por lo que estos indicadores han sido utilizados como 
parámetros de entrada, en la metodología de selección de tecnologías para las EDAR.

7. Las EDAR deben ubicarse en áreas alejadas de ecosistemas críticos y zonas recreativas, en un área segura, sin riesgo de deslizamiento, geológicamente inestables y sin riesgo de inundaciones, en áreas en el que los vientos regularmente no se desplacen hacia las poblaciones beneficiadas, así como que la descarga de las aguas residuales tratadas se realice a un efluente de flujo permanente o por infiltración al terreno.

8. La selección de una EDAR se basa en la consideración principalmente de tres atributos, que forman parte de los elementos propios de la idea y del perfil del proyecto de una EDAR, así como el aspecto operacional y de mantenimiento, los que se definen como, el atributo técnico, el económico y el ambiental.

9. La evaluación de las EDAR, no solo está limitada a la evaluación de aspectos cualitativos y cuantificativos, sino que está relacionada con el factor espacial, dado que involucra a las poblaciones aledañas, las áreas de vertimiento a los cuerpos de receptores de agua, así como el impacto ambiental que puede crear la mala disposición de los residuos.

\section{Conclusiones}

A modo de conclusión se considera importante resaltar que:

- La selección de la tecnología a utilizarse en las plantas depuradoras de aguas residuales, debe estar acoplada con las concepciones ambientales, económicas y de sostenibilidad a largo plazo, como componente esencial para el cumplimiento de los parámetros establecidos por la entidad reguladora para la protección del medio ambiente y la calidad vida en el planeta.

- Por otra parte, cabe destacar que dentro de las soluciones más efectivas y con mejor rendimiento se debe evaluar el proyecto de Biodiscos debido a su alto grado de efectividad tecnológica y su contribución a la solución práctica. Una de las grandes ventajas, es que, como la construcción de este tipo de plantas es modular, tiene una gran facilidad para ampliar las instalaciones y así tener una mayor eficiencia

- La selección de las tecnologías de depuración de aguas residuales apropiada para la ciudad de Ambato ha sido escogida en función de características propias de la zona, características del agua residual y del suelo, resultando así las tres alternativas propuestas en el proyecto.

\section{Referencias Bibliográficas}

Andreozzi, R., Raffele, M., Nicklas, P., Pharmaceuticals in STP effluents and solar photodegradation in aquatic environment. Chemosphere, n. 50, p. 1319-1330, 2003. http://dx.doi.org/10.1016/S0045-6535(02)00769-5.

APHA., (2009). Handbook of Water and Wastewater Treatment Plant Operations, Second Edition. USA: American Public Health Association. 
Benujah, B., (2013). Site Suitability Evaluation For Sewage Treatment Plant In Nagercoil Municipality, Tamil Nadu Using Remote Sensing Techniques.

Bdour, A., (2009). Perspectives on sustainable wastewater treatment technologies and reuse options in the urban areas of the Mediterranean region. Desalination, 162174.

Crites, R., (2000). Small and descentralized wastewater management systems. New York: Mc Graw Hill.

Chung, J., (2013). Assessment and selection of best available technology (BAT) for wastewater facilities in the leather tanning and finishing industry. Resources, Conservation and Recycling, 32-37.

Decreto Ejecutivo 3516 Registro Oficial Suplemento 2 de 31-mar.-2003 Ultima modificación: 23-mar.-2015. Texto Unificado Legislación Secundaria, Medio Ambiente, Libro VI- Articulo 216. Página 80-Tratamiento de aguas residuales urbanas y rurales.

Gernaey, KV., (2004). Activated sludge wastewater treatment plant modelling and simulation: state of the art. Environmental Modelling \& Software, 763-783.

Guardino, R., González M (2012)- La sostenibilidad de los proyectos. Caso de Estudio: Depuración de aguas residuales. Departament de Projectes d'Enginyeria (fins octubre 2015) - Ponències/Comunicacions de congressos [197]. URI http://hdl.handle.net/2117/16985.

Hammer, MJ., (2012). Water and wastewater technology. USA: Prentice Hall.

Hernández, A., (1996). Depuración de aguas residuales. Madrid España: Servicio de publicaciones de escuela de ingenieros de caminos, canales y puerto.

Hong, YS., (2003). Evolutionary self-organising modelling of a municipal wastewater treatment plant. Water Research, 1199-1212

INEC (2015)- Estadística de Información ambiental económica en Gobiernos Autónomos Descentralizados Municipales, Agua y Alcantarillado.

Informe Mundial de las Naciones Unidas sobre el Desarrollo de los Recursos Hídricos 2017. AGUAS RESIDUALES EL RECURSO DESAPROVECHADO. Publicado en 2017 por la Organización de las Naciones Unidas para la Educación, la Ciencia y la Cultura, 7, place de Fontenoy, 75352 París 07 SP, Francia. ISBN 978-92-3300058-2.

Lanka: International Water Management Institute. 35p. (IWMI Research Report 127). ISSN 1026-0862 ISBN 978-92-9090-698-8.

Metcalf, \& Eddy., (2003). Wastewater engineering: treatment, disposal and reuse. 4 edition. New york: Mc Graw Hill.

Montes, I., (2015)- Optimización de una planta de tratamiento de aguas residuales industriales en una Rectificadora de Tanques. Tesis de grado. Universidad Politécnica Salesiana. Ecuador. 
Noyola, A., Morgan, J., Guereca, L., (2013)- Selección de Tecnologías para el tratamiento de aguas residuales municipales-Guía de apoyo para ciudades pequeñas y medianas. Universidad Nacional Autónoma de México. Primera edición: 2013. ISBN: 978-607-02-4822-1.

Ortega, E., Salas, J., Ferrer, Y., Sobrados, L., (2008)- La depuración de las aguas residuales en pequeñas poblaciones españolas- Centro de las nuevas tecnologías del agua. Sevilla. España. https://d1wqtxts1xzle7.cloudfront.net/45764971.

Petrovic, M., Díaz, A., Ventura, F., Barceló, D., Occurrence and removal of estrogenic short-chain ethoxy nonylphenolic compound and their halogenated derivatives during drinking water production. Environmental Science \& Technology, v. 27, p. 4442-4448, 2003. http://dx.doi.org/10.1021/es034139

Raschid-Sally, L., Jayakody, P. 2008. Drivers and characteristics of wastewater agriculture in developing countries: Results from a global assessment. Colombo, Sri

Rico, A., Arahuetes, H., Morote, A., (2016)- Depuración y Reutilización de aguas residuales regeneradas en las regiones de Murcia y Valencia. Universidad de Alicante. Servicio de Publicaciones. ISBN: 978-84-16724-03-1. DOI 10.14198Libro Homenaje Alfredo Morales 2016-52. URI http://dx.doi.org/10.14198/LibroHomenajeAlfredoMorales2016$\underline{52}$ | http://hdl.handle.net/10045/58800.

Registro Oficial Edición Especial 387 de 04-nov.-2015- REFORMA TEXTO UNIFICADO LEGISLACION SECUNDARIA, MEDIO AMBIENTE, LIBRO VI, Decreto Ejecutivo 3516. Anexo 1: norma de calidad ambiental y de descarga de efluentes al recurso agua.

Rodríguez, JP., García, C A., \& Pardo Pinzón, J. (2015). Selección de tecnologías para el tratamiento de aguas residuales municipales. Revista Tecnura. Vol 19 Nro 46, pp 149-164. doi:10.14483/udistrital. jour. tecnura.2015. 4.a12.

Rubio, A., Chica, E., Peñuela, G., (2013)- Procesos de tratamiento de aguas residuales para la eliminación de contaminantes orgánicos emergentes. - Rev. Ambient. Água vol. 8 n. 3 Taubaté - Sep. / Dec. 2013. doi: 10.4136/ambi-agua.1176. Revista Ambiente \& Agua- An interdisciplinary Journal pf Applied Sciencie. ISSN 1980993X - doi:10.4136/1980-993X.

Sánchez, J., (2017)- Saneamiento descentralizado y reutilización sustentable de las aguas residuales municipales en México. Soc. ambient. no.14 Lerma Campeche jul. 2017. versión On-line ISSN 2007-6576.

Schoenberger, H., (2009). Integrated pollution prevention and control in large industrial installations on the basis of best available techniques - The Sevilla Process. Journal of Cleaner Production, 1526-1529.

Telecomunicaciones y de Ingeniería de Sistemas, Escuela de Ingeniería, Universidad Autónoma de Barcelona, 08193 Bellaterra, Barcelona, Spain. Revista 
Iberoamericana de Automática e Informática industrial 14 (2017) 217-233. DOI: https://doi.org/10.1016/j.riai.2017.05.004. https://doi.org/10.4995/riai.

Vázquez, M., (2017), Contaminación del agua: causas, consecuencias y soluciones: https://agua.org.mx/contaminacion-del-agua-causas-consecuencias-soluciones/.

Vilanova, R., Sant, I., Pedret, C., (2017)- Control y Operación de Estaciones Depuradoras de Aguas Residuales: Modelado y Simulación. Departamento de

Villaseñor, J. (2001). Eliminación biológica de fósforo en aguas residuales. Castilla: Universidad de Castilla

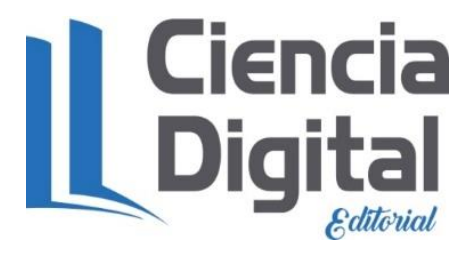


PARA CITAR EL ARTÍCULO INDEXADO.

Ortiz Montero, S. A., Núnez Aldás, G. W., López Arboleda, A. G., \& Frías Torres, A. X. (2021). La depuración de aguas residuales. Estudios de soluciones para el municipio de $\begin{array}{llll}\text { Ambato. } & \text { Ecuador. } & \text { 250-270. }\end{array}$ https://doi.org/10.33262/concienciadigital.v4i3.1803

\section{Ciencia}

El artículo que se publica es de exclusiva responsabilidad de los autores y no necesariamente reflejan el pensamiento de la Revista Conciencia Digital.

El artículo queda en propiedad de la revista y, por tanto, su publicación parcial y/o total en otro medio tiene que ser autorizado por el director de la Revista Conciencia Digital.

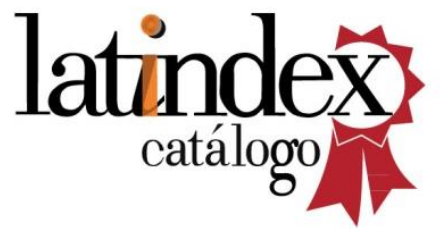

